$192+/-88)$. As UR Surgeries increased to $>50 \%$ of overall number of surgeries for advanced disease, the mean OT was a full operating day $(2018 ; 367 \pm 132,2019 ; 375 \pm 153$ and $2020 ; 426 \pm 122)$. Further increases in UR rates had less impact on OT $(\mathrm{F}(3,62)=1.11, \mathrm{p}>0.05)$.

Conclusion* Effectively treating AOC patients requires appropriate theatre resources. We recommend that the standard of care for cytoreductive surgeries operated with maximal effort should be that only case is performed on that day. Funding and resource allocation for ovarian cancer surgery in the United Kingdom needs to reflect the greater theatre utilisation needed for these patients.

\section{ROLE OF OPTIMAL CYTOREDUCTION IN ADVANCED STAGE MALIGNANT OVARIAN GERM CELL TUMORS, DATA FROM THE MITO-9 TRIAL}

${ }^{1} \mathrm{~A}$ Bergamini*, ${ }^{2} \mathrm{C}$ Cassani, ${ }^{3} \mathrm{~F}$ Raspagliesi, ${ }^{3} \mathrm{G}$ Bogani, ${ }^{1} \mathrm{~L}$ Bocciolone, ${ }^{1} \mathrm{E}$ Rabaiotti, ${ }^{1} \mathrm{R}$ Cioffi, ${ }^{1} \mathrm{G}$ Sabetta, ${ }^{4} \mathrm{G}$ Scarfone, ${ }^{5} \mathrm{~A}$ Savarese, ${ }^{6} \mathrm{MG}$ Ferrandina, ${ }^{6} \mathrm{~F}$ Mascilini, ${ }^{7} \mathrm{G}$ Cormio, ${ }^{8,9} \mathrm{G}$ Giorda, ${ }^{10} \mathrm{~S}$ Pignata, ${ }^{1} \mathrm{G}$ Taccagni, ${ }^{1} \mathrm{G}$ Mangili. ${ }^{1}$ IRCCS San Raffaele Hospital, Obstetrics and Gynecology, Milan; ${ }^{2}$ Fondazione I. R.C.C.S. Policlinico San Matteo, Pavia, Italy; ${ }^{3}$ stituto Nazionale dei Tumori | Fondazione IRCCS, Milano, Italy; ${ }^{4}$ Mangiagalli Clinic IRCCS Cà Granda Foundation Ospedale Maggiore Policlinico, Milano, Italy; ${ }^{5}$ Ospedale Regina Elena, Roma, Italy; ${ }^{6}$ Agostino Gemelli University Policlinic, Roma, Italy; ${ }^{7}$ Ospedale Oncologico Giovanni Paolo II, Bari, Italy; ${ }^{8}$ Aviano, Italy; ${ }^{9}$ Oncology Referral Center, Aviano, Italy; ${ }^{10}$ IRCCS Istituto Nazionale Tumori "Fondazione G. Pascale"

10.1136/ijgc-2021-ESGO.469

Introduction/Background" the role of optimal cytoreduction in advanced stage MOGCTs is still a debated issue, considering the high chemosensitivity of these tumors and young patients' age. The aim of the present study was to analyze the role of residual tumor at primary surgery in advanced stage MOGCTs.

Methodology clinicopathological data from patients with stage III-IV MOGCTs were retrospectively collected among MITO centers (Multicenter Italian Trials in Ovarian cancer and gynecologic malignancies) and analyzed. All patients underwent primary surgery. Optimal cytoreduction was defined as macroscopic residual tumor $=0$. All patients received adjuvant platinum-based chemotherapy. Clinicopathological features were evaluated for association with relapse.

Result(s)* 42 patients were included. 18 (42.8\%) were affected by dysgerminomas, $24(57.2 \%)$ by non-disgerminomatous MOGCTs. 24 patients (57.2\%) achieved optimal cytoreduction at primary surgery (residual tumor $=0$ ). No statistical difference was detected in stage distribution, age, rate of optimal cytoreduction between the dysgerminomas and non dysgerminomatous groups. Median 5year- disease free survival (DFS) rates were $87.8 \%$ and $50.0 \%$ for dysgerminomas and non-dysgerminomas, respectively. In the whole cohort, there was no difference in terms of 5 years-DFS according to residual tumor at primary surgery $(74.6 \%$ vs $56.0 \%, \mathrm{p}=0.25)$. Residual tumor at primary surgery was a prognostic factor for DFS only for non-dysgerminomatous MOGCTs (64\% vs 30\% for optimal vs suboptimal cytoreduction, respectively, $\mathrm{p}=0.05)$.

Conclusion* in the present study, optimal cytoreduction at primary surgery was a prognostic factor affecting disease-free survival only for non-disgerminomatous MOGCTs.

\section{IS THE SEROUS TUBAL INTRAEPITHELIAL CARCINOMA TO BLAME FOR PERITONEAL CARCINOMATOSIS AFTER RISK-REDUCING SALPINGO-OOPHORECTOMY?}

${ }^{1} M P$ Steenbeek*, ${ }^{2} \mathrm{~J}$ Bulten, 'S Study Group, ${ }^{3}$ RPMG Hermens, ${ }^{3} \mathrm{I}$ Inthout, ${ }^{1} \mathrm{JA}$ De Hullu. ${ }^{1}$ Radboud university medical center, Obstetrics and Gynaecology, Nijmegen, Netherlands; ${ }^{2}$ Radboud university medical center, Pathology, Nijmegen, Netherlands; ${ }^{3}$ Radboud university medical center, Scientific Institute for Quality of Healthcare, Nijmegen, Netherlands

\subsection{6/ijgc-2021-ESG0.470}

Introduction/Background* After risk-reducing salpingo-oophorectomy (RRSO), there is an approximate 3.9\% and $1.9 \%$ risk of developing peritoneal carcinomatosis (PC) for BRCA1/2

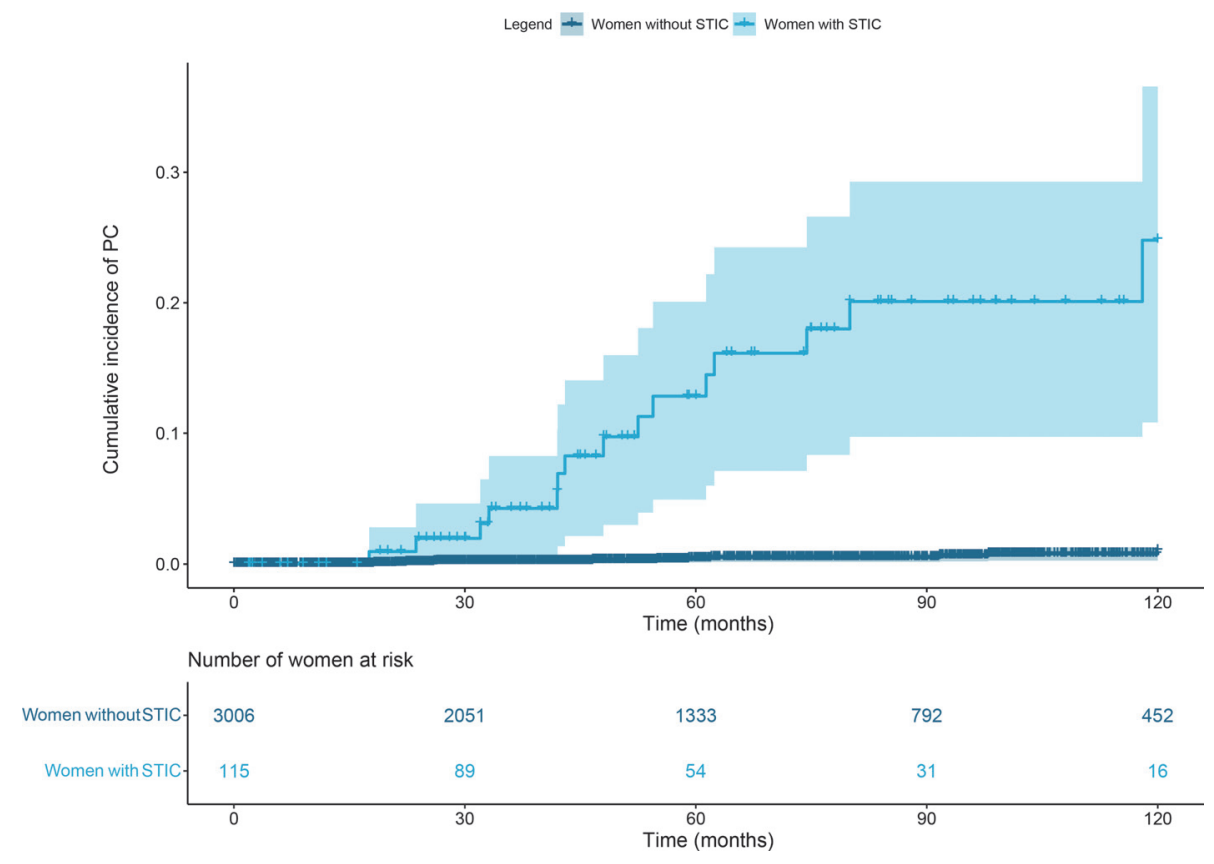

Abstract 781 Figure 1 The incidence of PC in women with and without STIC at RRSO. 